PAPER

\section{Hamiltonian positivity of massive spin-2 particles} via a rank-2 tensor

To cite this article: D Benndorf et al 2017 Class. Quantum Grav. 34045008

View the article online for updates and enhancements.

\section{Related content}

- A note on the ubiquity of the traceless spin
$\frac{\text { connection in dual massive spin-2 theories }}{\text { D Dalmazi }}$
- Recent developments in bimetric theory
Angnis Schmidt-May and Mikael von
Strauss
- Massive spin-2 particles via embedment of
the Fierz-Pauli equations of motion
D Dalmazi, A L R dos Santos and E L
Mendonça

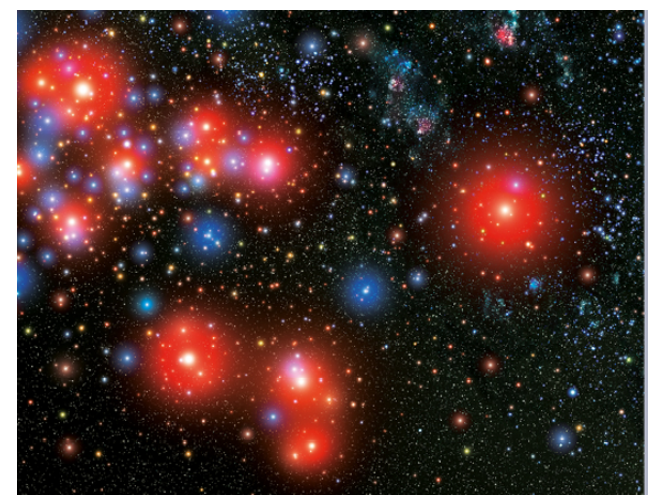

A.A IOP Astronomy ebooks

Part of your publishing universe and your first choice for astronomy, astrophysics, solar physics and planetary science ebooks. iopscience.org/books/aas 


\title{
Hamiltonian positivity of massive spin-2 particles via a rank-2 tensor
}

\author{
D Benndorf, D Dalmazi and A L R dos Santos \\ UNESP — Campus de Guaratinguetá—DFQ Avenida Dr. Ariberto Pereira da Cunha, \\ 333 CEP 12516-410 — Guaratinguetá-SP-Brazil \\ E-mail: dbenndorf@yahoo.com.br, dalmazi@feg.unesp.br \\ and alessandroribeiros@yahoo.com.br
}

Received 8 September 2016, revised 31 December 2016

Accepted for publication 16 January 2017

Published 31 January 2017

\begin{abstract}
There are three families of Lagrangians describing massive spin-2 particles via a general (nonsymmetric) rank-2 tensor. Each of those families depends on an arbitrary real parameter, one of them includes the paradigmatic FierzPauli theory whose Hamiltonian positivity is known and reviewed here. Here we apply the plain Dirac-Bergmann procedure in the two remaining families. We identify all Hamiltonian constraints and prove both positivity of the reduced Hamiltonian and correct counting of degrees of freedom. The positivity of each spin mode contribution is demonstrated by using spin projection operators. The massless cases are also examined. In particular, we prove positivity of the reduced Hamiltonian and correct counting of degrees of freedom of a Weyl invariant description of massless spin-2 particles.
\end{abstract}

Keywords: massive gravity, Fierz-Pauli, massive spin-2, constraints

\section{Introduction}

The recent detection of gravitational waves [1] remarkably confirms another prediction of general relativity (GR). Even though GR can be understood from the field theory point of view as a self-interacting theory for massless spin-2 particles, the existence of massive gravitons is not totally ruled out by experiments. The results of [1] set an upper bound about $10^{-22} \mathrm{ev}$ for the graviton mass [2]. From the theoretical standpoint the existence of massive gravitons has been investigated in the past in, for instance, [3-6]. Two difficult problems raised up at that time. Namely, the discontinuity of the massless limit (vDVZ mass discontinuity) already present in the linearized theory $[3,4]$ and the appearance of a nonphysical (ghost) degree of freedom in the nonlinear theory [5].

The first problem can be solved by taking into account nonlinear terms along the lines of [6], see also [7] and the review works [8, 9], however it leads us back to the ghost problem. 
Quite recently, motivated also by the problem of the large distance acceleration of the universe $[10,11]$, the authors of [12] have suggested a massive gravity theory where both problems have been tackled via a judicious choice of a nonlinear potential for the metric fluctuation $h_{\mu \nu}$. The same potential has been used in the phenomenologically more interesting bimetric model of [13].

The potential suggested in [12] is built up on the top of the Fierz-Pauli (FP) theory [14] which is the linearized (free) limit of the massive gravity model of [12]. One might wonder what happens if we replace the FP theory as our paradigm for free massive spin-2 particles. Which features of the recent theories of massive gravity are model independent? Regarding that point, first we must know which theories can replace the FP model.

If we start with an arbitrary (nonsymmetric) rank-2 tensor $e_{\mu \nu}$ it is possible to show from first principles, see [15] for a recent review on that point, that the so called FP conditions must be satisfied. In particular, the trace $\eta_{\mu \nu} \mathrm{e}^{\mu \nu}$ and the antisymmetric components $e_{[\mu \nu]}=\left(e_{\mu \nu}-e_{\nu \mu}\right) / 2$ must vanish. However, we are not able to describe massive spin-2 particles if we have a traceless symmetric tensor from the start. Auxiliary fields are needed to produce the required amount of constraints. The FP theory is somehow a minimum theory where the trace, which vanishes on shell, is the only auxiliary field, but there are other possibilities, see for example the recent work [16] for a Weyl invariant massive spin-2 model.

In [17] we have made a rather general Ansatz for a Lagrangian $\mathcal{L}\left(e_{\mu \nu}\right)$ with at most two derivatives in $D=3+1$. Demanding that the propagator contains only one massive pole, with positive residue, in the spin-2 sector, we have found three families of Lagrangians describing massive spin-2 particles. Each of the families depends upon an arbitrary real constant. The first family contains the FP model. Its arbitrary constant ' $d$ ' lies in the mass term which contains a term like $d e_{[\mu \nu]}^{2}$, the FP model corresponds to $d=0$. The second family we call nFP (non Fierz-Pauli) since the mass term $e_{\mu \nu} \mathrm{e}^{\nu \mu}+c e^{2}$ contains an arbitrary real constant $c$ which does not need to be fixed like the FP choice $(c=-1)$. In the third model $\mathcal{L}\left(a_{1}\right)$, the arbitrariness is present in the derivative terms, see (47), it includes the model suggested in [18], which corresponds to $a_{1}=-1 / 4$.

We have shown in [17], in a purely Lagrangian approach, that the FP conditions and the Klein-Gordon equation are satisfied for all three families. Since the Hamiltonian approach plays a key role in the proof of absence of ghosts in massive gravity models, see e.g. $[19,20]$ and the review work [9], if we hope to develop a nonlinear massive gravity theory on the top of the new families of massive spin-2 models we better set the stage for future calculations. Here we confirm the consistency of those models in the Hamiltonian formalism by proving Hamiltonian positivity and correct counting of degrees of freedom. For sake of comparison with the alternative families $\mathcal{L}_{\mathrm{nFP}}$ and $\mathcal{L}\left(a_{1}\right)$ and in order to present our general approach based on spin projection operators, we review in section 2 the Hamiltonian formalism applied in the usual FP theory for both massless (linearized Einstein-Hilbert (LEH)) and massive cases. In sections 3 and 4 we work out the $\mathcal{L}_{\text {nFP }}$ and $\mathcal{L}\left(a_{1}\right)$ cases. In section 5 we present our conclusions. In appendix we have an appendix with basic formulae for spin projection operators.

\section{Review of the Fierz-Pauli theory}

We begin by considering the FP theory which describes massive and massless (if $m=0$ ) spin-2 particles [14]. The Hamiltonian analysis of the FP theory via Dirac-Bergmann algorithm [21-23] has been carried out, for instance, in [24] (massive and massless cases), see also the recent work [25] where the positivity of each spin helicity mode is shown via 
a first order formalism. In this section we establish our formalism and our notation. We prove the positivity of the FP Hamiltonian in the massless and massive case by means of spin projection operators given in the appendix. Our analysis holds in arbitrary dimensions $D \geqslant 3$.

\subsection{The massless case}

The massless FP theory is equivalent to the linearized Einstein-Hilbert (LEH) theory. It is given by

$$
\mathcal{L}_{\mathrm{LEH}}=-\frac{1}{4} \partial_{\mu} h_{\alpha \beta} \partial^{\mu} h^{\alpha \beta}+\frac{1}{4} \partial_{\mu} h\left(\partial^{\mu} h-2 \partial_{\nu} h^{\nu \mu}\right)+\frac{1}{2} \partial_{\mu} h^{\mu \nu} \partial^{\alpha} h_{\alpha \nu},
$$

where $h_{\mu \nu}, \mu=0,1, \cdots, D-1$, is a symmetric tensor field and $h=\eta^{\mu \nu} h_{\mu \nu}$. Throughout this work we use $\eta_{\mu \nu}=(-,+, \cdots,+)$. This model is invariant under linearized reparametrizations $\delta h_{\mu \nu}=\partial_{\mu} \xi_{\nu}+\partial_{\nu} \xi_{\mu}$. We have $D$ primary constraints

$$
\phi^{0 \mu}=\pi^{0 \mu}=\frac{\partial \mathcal{L}_{\mathrm{LEH}}}{\partial \dot{h}_{0 \mu}} \approx 0
$$

where $\phi^{0 \mu} \approx 0$ stands for Dirac's weakly zero condition, i.e. we first calculate all necessary Poisson brackets before we set the constraints strongly to zero. From the canonical momenta

$$
\pi^{i j}=\frac{\partial \mathcal{L}_{\mathrm{LEH}}}{\partial \dot{h}_{i j}}=\frac{1}{2} \dot{h}_{i j}-\frac{1}{2} \delta_{i j} \dot{\bar{h}}+\delta_{i j} \partial_{k} h_{0 k}-\frac{1}{2} \partial_{i} h_{0 j}-\frac{1}{2} \partial_{j} h_{0 i},
$$

where $\bar{h}=\delta_{i j} h_{i j}$, we can obtain $\dot{h}_{i j}$ in terms of $\pi^{i j}$. The primary hamiltonian which incorporates the primary constraints (2) via Lagrange multipliers $\lambda_{\mu}$ is given by

$$
H_{P}=\int \mathrm{d}^{D-1} x\left[\pi^{\mu \nu} \dot{h}_{\mu \nu}-\mathcal{L}_{\mathrm{LEH}}+\lambda_{\mu} \phi^{0 \mu}\right]
$$

The consistency conditions $\dot{\phi}^{0 \mu} \approx 0$ lead to the secondary constraints

$$
\begin{aligned}
& \chi^{0}=\nabla^{2} \bar{h}-\partial_{i} \partial_{j} h_{i j} \approx 0, \\
& \chi^{i}=\partial_{j} \pi^{i j} \approx 0,
\end{aligned}
$$

which are trivially preserved $\dot{\chi}^{\mu} \approx 0$ without introducing new constraints. All Lagrange multipliers $\lambda_{\mu}$ remain undetermined. So the LEH theory has $2 D$ first class constraints. Thus, one has $D(D+1) / 2-2 D=D(D-3) / 2$ degrees of freedom which correspond to the two helicities +2 and -2 in $D=4$.

In order to verify the positivity of the reduced Hamiltonian, we begin by using the constraints as strong equalities in (4) which reduces the primary Hamiltonian to the canonical one and removes $h_{0 \mu}$ from the Hamiltonian. The partially reduced Hamiltonian is given by

$$
H^{(p r)}=\int \mathrm{d}^{D-1} x\left\{\pi^{i j} \pi^{i j}-\frac{\bar{\pi} \bar{\pi}}{(D-2)}+\frac{1}{4} \partial_{i} h_{j k} \partial_{i} h_{j k}-\frac{1}{4} \partial_{i} \bar{h}\left(\partial_{i} \bar{h}-2 \partial_{k} h_{i k}\right)-\frac{1}{2} \partial_{i} h_{i j} \partial_{k} h_{k j}\right\}
$$

where $\bar{\pi}=\delta_{i j} \pi^{i j}$. Bilinear contractions of rank-2 tensors and derivatives, as in $H^{(p r)}$, can always be written in terms of spin projection and transition operators as shown in the appendix. Using the formulae in the appendix we have 


$$
\begin{aligned}
H^{(p r)}= & \int \mathrm{d}^{D-1} x\left\{\pi^{i j}\left[P_{S S}^{(2)}+P_{S S}^{(1)}+\frac{(D-3)}{(D-2)} P_{W W}^{(0)}-\frac{\left(P_{S W}^{(0)}+P_{W S}^{(0)}\right)}{\sqrt{D-2}}\right]^{i j, k l} \pi^{k l}\right. \\
& \left.+h_{i j}\left[-\frac{\nabla^{2}}{4} P_{S S}^{(2)}-\frac{(D-1)}{4} \nabla^{2} P_{S S}^{(0)}-\frac{\sqrt{D-2}}{4} \nabla^{2}\left(P_{S W}^{(0)}+P_{W S}^{(0)}\right)\right]^{i j, k l} h_{k l}\right\},
\end{aligned}
$$

where the spin-s operators $P_{A B}^{(s)}$ are displayed in the appendix. From the constraints $\chi^{\mu}=0$ we obtain $\theta^{i j} h_{i j}=0$ and $\omega_{i j} \pi^{i j}=0$, where $\theta_{i j}=\delta_{i j}-\omega_{i j}$ is a spin-1 projection operator, and $\omega_{i j}=\partial_{i} \partial_{i} / \nabla^{2}$ is a spin-0 projection operator. Consequently, we get rid of the $s=1$ and $s=0$ operators in (8) and end up with the reduced Hamiltonian given only in terms of the spin-2 projection operators as expected for massless gravitons ( \pm 2 helicities ):

$$
H^{(r)}=\int \mathrm{d}^{D-1} x\left\{\pi_{i j}\left(P_{S S}^{(2)}\right)^{i j, k l} \pi_{k l}+h_{i j}\left(-\frac{\nabla^{2}}{4} P_{S S}^{(2)}\right)^{i j, k l} h_{k l}\right\} .
$$

Now a comment is in order, whenever we have an arbitrary projection operator $P_{A B}^{(s)}$ sandwiched among two equal tensors we have a positive definite bilinear, because

$$
\pi_{i j}\left(P_{A B}^{(s)}\right)^{i j, k l} \pi_{k l}=\pi_{i j}\left(P_{A B}^{(s)}\right)^{i j}{ }_{m n}\left(P_{A B}^{(s)}\right)^{m n, k l} \pi_{k l}=\left[\left(P_{A B}^{(s)}\right)^{m n, k l} \pi_{k l}\right]^{2} \geqslant 0 .
$$

Since $-\nabla^{2}$ has only positive eigenvalues, it is clear that (9) is positive definite (classical stability).

\subsection{The $m \neq 0$ case}

The massive FP model [14] is written in the form

$$
\mathcal{L}_{\mathrm{FP}}=\mathcal{L}_{\mathrm{LEH}}-\frac{m^{2}}{4}\left(h_{\mu \nu} h^{\mu \nu}-h^{2}\right)
$$

where $\mathcal{L}_{\text {LEH }}$ is given by (1). The mass term breaks the invariance $\delta h_{\mu \nu}=\partial_{\mu} \xi_{\nu}+\partial_{\nu} \xi_{\mu}$. The canonical momenta are unaffected by the mass term in (11). Thus, we still have $D$ primary constraints $\phi^{0 \mu}=\pi^{0 \mu} \approx 0$. From $\dot{\phi}^{0 \mu} \approx 0$ we obtain the secondary constraints

$$
\begin{aligned}
& \chi^{0}=\left(\nabla^{2}-m^{2}\right) \bar{h}-\partial_{i} \partial_{j} h_{i j} \approx 0, \\
& \chi^{i}=\partial_{j} \pi^{i j}+\frac{m^{2}}{2} h_{0 i} \approx 0 .
\end{aligned}
$$

The constraints $\dot{\chi}^{0} \approx 0$ and $\ddot{\chi}^{0} \approx 0$ lead to tertiary and quaternary constraints respectively

$$
\begin{aligned}
& \zeta^{0}=-\frac{m^{2} \bar{\pi}}{(D-2)}+\frac{m^{2}}{2} \partial_{i} h_{0 i} \approx 0, \\
& \Omega^{0}=\frac{m^{4}}{2} \frac{(D-1)}{(D-2)}\left(h_{00}-\bar{h}\right) \approx 0,
\end{aligned}
$$

while $\lambda_{0 i}$ and $\lambda_{00}$ are determined by $\dot{\chi}^{i} \approx 0$ and $\dot{\Omega}^{0} \approx 0$ respectively. So the massive case has $2(D+1)$ second class constraints. The number of degrees of freedom is $(D+1)(D-2) / 2$, corresponding to massive spin-2 particles $2 s+1=5$ in $D=4$. Using the constraints as strong equalities we eliminate $h_{0 \mu}$. The partially reduced Hamiltonian is given by 


$$
\begin{aligned}
H^{(p r)}= & \int \mathrm{d}^{D-1} x\left\{\pi^{i j} \pi^{i j}-\frac{\bar{\pi} \bar{\pi}}{(D-2)}+\frac{2}{m^{2}} \partial_{i} \pi^{i j} \partial_{k} \pi^{k j}+\frac{1}{4} \partial_{i} h_{j k} \partial_{i} h_{j k}+\frac{1}{4} \partial_{i} \bar{h} \partial_{i} \bar{h}\right. \\
& \left.-\frac{1}{2} \partial_{i} h_{i j} \partial_{k} h_{k j}+\frac{m^{2}}{4} h_{i j} h_{i j}+\frac{m^{2}}{2} \bar{h} \bar{h}\right\} .
\end{aligned}
$$

The constraints (12)-(14) make the terms containing the transition operators proportional to the projection operator $P_{W W}^{(0)}$. Finally, the reduced Hamiltonian becomes a sum of spin2 , spin-1 and spin-0 contributions as expected for a massive spin-2 particle with five degrees of freedom $( \pm 2, \pm 1,0)$. Each helicity contribution is separately positive definite, see (10),

$$
\begin{aligned}
H^{(r)}= & \int \mathrm{d}^{D-1} x\left\{\pi^{i j}\left[P_{S S}^{(2)}+\frac{\left(m^{2}-\nabla^{2}\right)}{m^{2}} P_{S S}^{(1)}+\frac{(D-1)}{(D-2)} P_{W W}^{(0)}\right]^{i j, k l} \pi^{k l}\right. \\
& \left.+h_{i j}\left[\frac{\left(m^{2}-\nabla^{2}\right)}{4} P_{S S}^{(2)}+\frac{m^{2}}{4} P_{S S}^{(1)}+\frac{(D-1)}{4}\left(m^{2}-\nabla^{2}\right) P_{S S}^{(0)}\right]^{i j, k l} h_{k l}\right\} .
\end{aligned}
$$

\section{The $\mathcal{L}_{\text {nFP }}$ model}

The nFP model describes massive spin-2 particles in terms of a nonsymmetric tensor $e_{\mu \nu}$. In $D=4$, the analytic structure of the propagator has been analyzed in [26], where it is shown to be ghost-free. Although there is no local field redefinition relating the nFP and the usual FP model, after the introduction of an extra (spectator) scalar field it is possible to show that they are dual to each other [27]. There is a dual map between correlation functions in both models. In its massless form the model describes massless spin- 2 particles and first appeared in [28] while for the massive case it appeared in [26], both in $D=4$, for the $D$ - dimensional generalization see [27]. Next we start with the massless case.

\subsection{The $m=0$ case}

The massless version of the $\mathcal{L}_{\mathrm{nFP}}$ model in $D$ dimensions is given by $[27,28]$

$$
\begin{aligned}
\mathcal{L}_{\mathrm{nFP}}= & -\frac{1}{4} \partial_{\mu} e_{(\alpha \beta)} \partial^{\mu} \mathrm{e}^{(\alpha \beta)}+\frac{1}{4(D-1)} \partial_{\mu} e\left[\partial^{\mu} e-2 \partial_{\nu} \mathrm{e}^{\nu \mu}\right]+\frac{1}{2}\left[\partial_{\mu} \mathrm{e}^{(\mu \nu)}\right]^{2} \\
& -\frac{1}{4} \frac{(D-2)}{(D-1)}\left[\partial_{\mu} \mathrm{e}^{\mu \nu}\right]^{2},
\end{aligned}
$$

where we are using the notation $e_{(\mu \nu)}=\left(e_{\mu \nu}+e_{\nu \mu}\right) / 2$ for the symmetric part of $e_{\mu \nu}$. The antisymmetric part will be represented by $e_{[\mu \nu]}=\left(e_{\mu \nu}-e_{\nu \mu}\right) / 2$. The model (18) describes massless spin-2 particles and is invariant under the linearized reparametrizations, Weyl transformations and transverse antisymmetric shifts $\delta e_{\mu \nu}=\partial_{\nu} \xi_{\mu}+\eta_{\mu \nu} \Lambda+\partial^{\alpha} \Lambda_{\alpha \mu \nu}$, where $\Lambda_{\alpha \mu \nu}$ is a totally antisymmetric tensor. Its particle content has been figured out in [26] from the Lagrangian point of view and from the analytic structure of the propagator.

We start the Hamiltonian analysis by splitting the Lagrangian density (18) in time and space components. Up to total derivatives, we have 


$$
\begin{aligned}
\mathcal{L}_{\mathrm{nFP}}= & \frac{1}{4} \dot{e}_{(i j)} \dot{e}_{(i j)}-\frac{1}{4(D-1)} \dot{\bar{e}} \dot{\bar{e}}-\frac{1}{4} \frac{(D-2)}{(D-1)} \dot{e}_{0 i} \dot{e}_{0 i}+\frac{1}{(D-1)} \dot{\bar{e}}_{j} \partial_{(0 j)} \\
& +\frac{1}{4(D-1)} \dot{e}_{0 i}\left[2(D-2) \partial_{i} e_{00}+(D-3) \partial_{j} e_{j i}-(D-1) \partial_{j} e_{i j}\right]-\frac{1}{2} \dot{e}_{(i j)} \partial_{i} e_{j 0} \\
& -\frac{1}{4(D-1)} \partial_{i} e_{00}\left[(D-2) \partial_{i} e_{00}+2 \partial_{i} \bar{e}-2 \partial_{j} e_{i j}\right]+\frac{1}{2} \partial_{i} e_{(0 j)} \partial_{i} e_{(0 j)} \\
& +\frac{1}{4(D-1)} \partial_{i} \bar{e}\left[\partial_{i} \bar{e}-2 \partial_{k} e_{i k}\right]-\frac{1}{4} \partial_{i} e_{(j k)} \partial_{i} e_{(j k)}+\frac{1}{8} \frac{(D-3)}{(D-1)} \partial_{i} e_{i 0} \partial_{j} e_{j 0} \\
& -\frac{1}{8} \partial_{i} e_{0 i}\left[\partial_{j} e_{0 j}+2 \partial_{j} e_{j 0}\right]-\frac{1}{8} \frac{(D-3)}{(D-1)} \partial_{i} e_{i j} \partial_{k} e_{k j}+\frac{1}{8} \partial_{i} e_{j i}\left[\partial_{k} e_{j k}+2 \partial_{k} e_{k i}\right]
\end{aligned}
$$

where $\bar{e}=\delta_{i j} e_{i j}$. The canonical momenta are defined by $\pi^{\mu \nu}=\delta \mathcal{L}_{\mathrm{nFP}} / \delta \dot{e}_{\mu \nu}$. So

$$
\begin{aligned}
& \pi^{\mu 0}=0, \quad \pi^{[i j]}=0, \quad \bar{\pi}=\delta_{i j} \pi^{i j}=0, \\
& \pi^{0 i}=-\frac{1}{2} \frac{(D-2)}{(D-1)} \dot{e}_{0 i}+\frac{1}{2} \frac{(D-2)}{(D-1)} \partial_{i} e_{00}+\frac{1}{4} \frac{(D-3)}{(D-1)} \partial_{j} e_{j i}-\frac{1}{4} \partial_{j} e_{i j}, \\
& \pi^{(i j)}=\frac{1}{2} \dot{e}_{(i j)}-\frac{1}{2(D-1)} \delta_{i j} \dot{\bar{e}}+\frac{1}{(D-1)} \delta_{i j} \partial_{k} e_{(0 k)}-\frac{1}{4} \partial_{i} e_{j 0}-\frac{1}{4} \partial_{j} e_{i 0} .
\end{aligned}
$$

Therefore we have the following primary constraints

$$
\begin{gathered}
\phi^{\mu 0}=\pi^{\mu 0} \approx 0, \\
\phi^{[i j]}=\pi^{[i j]} \approx 0, \\
\bar{\phi}=\bar{\pi}-\frac{1}{2} \partial_{i} e_{0 i} \approx 0 . \\
\mathcal{H}_{\mathrm{nFP}}=\pi^{(i j)} \pi^{(i j)}-\frac{(D-1)}{(D-2)} \pi^{0 i} \pi^{0 i}+\pi^{0 i}\left[\partial_{i} e_{00}+\frac{1}{2} \frac{(D-3)}{(D-2)} \partial_{j} e_{j i}-\frac{1}{2} \frac{(D-1)}{(D-2)} \partial_{j} e_{i j}\right] \\
+\pi^{(i j)} \partial_{i} e_{j 0}-\frac{1}{4(D-1)} \partial_{i} \bar{e}\left[\partial_{i} \bar{e}-2 \partial_{i} e_{00}-2 \partial_{k} e_{i k}\right]+\frac{1}{4} \partial_{i} e_{(j k)} \partial_{i} e_{(j k)} \\
+\frac{1}{16} \frac{(D-3)}{(D-2)} \partial_{i} e_{i j} \partial_{k} e_{k j}-\frac{1}{8} \frac{(D-1)}{(D-2)} \partial_{i} e_{i j} \partial_{k} e_{j k}-\frac{1}{16} \frac{(3 D-5)}{(D-2)} \partial_{i} e_{j i} \partial_{k} e_{j k} \\
+\frac{1}{8} \frac{(D-3)}{(D-1)} \partial_{i} e_{0 i}\left[\partial_{j} e_{0 j}+2 \partial_{j} e_{j 0}\right]-\frac{1}{8} \partial_{i} e_{0 j}\left[\partial_{i} e_{0 j}+2 \partial_{i} e_{j 0}\right] .
\end{gathered}
$$

Adding the primary constraints to the canonical Hamiltonian $H_{\mathrm{nFP}}=\int \mathrm{d}^{D-1} x \mathcal{H}_{\mathrm{nFP}}$ we find the primary Hamiltonian

$$
H_{P}=\int \mathrm{d}^{D-1} x\left\{\mathcal{H}_{\mathrm{nFP}}+\lambda_{\mu 0} \phi^{\mu 0}+\lambda_{[i j]} \phi^{[i j]}+\bar{\lambda} \bar{\phi}\right\}
$$

If we apply the consistency conditions $\dot{\phi}^{00} \approx 0, \dot{\phi}^{i 0} \approx 0$ and $\dot{\phi}^{[i j]} \approx 0$, we find the following secondary constraints respectively 


$$
\begin{aligned}
& \chi^{0}=\partial_{i} \pi^{0 i}+\frac{1}{2(D-1)} \nabla^{2} \bar{e} \approx 0, \\
& \chi^{i}=\partial_{j} \pi^{(i j)}+\frac{1}{4} \frac{(D-3)}{(D-1)} \partial_{i} \partial_{j} e_{0 j}-\frac{1}{4} \nabla^{2} e_{0 i} \approx 0, \\
& \chi^{[i j]}=\partial_{i}\left[\pi^{0 j}+\frac{1}{2} \partial_{k} e_{(j k)}\right]-\partial_{j}\left[\pi^{0 i}+\frac{1}{2} \partial_{k} e_{(i k)}\right] \approx 0 .
\end{aligned}
$$

The equation $\dot{\bar{\phi}} \approx 0$ does not give a new constraint.

When we apply the consistency condition on the secondary constraints, we verify that no tertiary constraints are introduced as well as no Lagrange multipliers are determined. Then, the process ends up. Therefore, the massless $\mathcal{L}_{\mathrm{nFP}}$ theory, described by $\left(e_{\mu \nu}, \pi_{\mu \nu}\right)$ has $D(D+3) / 2$ constraints ${ }^{1}$. They are all first class. So, one has $2 D^{2}-2 D(D+3) / 2$ Hamiltonian degrees of freedom which amounts to $D(D-3) / 2$ Lagrangian degrees of freedom. It is the same number of degrees of freedom of the massless FP theory in $D$ dimensions.

The constraints can be used to write the partially reduced hamiltonian as

$$
\begin{aligned}
H^{(p r)}= & \int \mathrm{d}^{D-1} x\left\{\pi^{(i j)} \pi^{(i j)}+\frac{(D-3)}{(D-1)^{2}} \bar{\pi} \bar{\pi}+\frac{2(D-3)}{(D-1)} \frac{\partial_{i} \bar{\pi} \partial_{k} \pi^{i k}}{\nabla^{2}}+2 \frac{\left[\partial_{i} \pi^{(i j)}\right]^{2}}{\nabla^{2}}\right. \\
& +\frac{1}{4} \partial_{i} e_{(j k)} \partial_{i} e_{(j k)}-\frac{1}{4(D-2)} \partial_{i} \bar{e}\left[\partial_{i} \bar{e}-2 \partial_{k} e_{i k}\right]-\frac{1}{2} \partial_{i} e_{(i j)} \partial_{k} e_{(k j)} \\
& \left.-\frac{1}{4} \frac{(D-3)}{(D-2)} \frac{\left[\partial_{i} \partial_{j} e_{(i j)}\right]^{2}}{\nabla^{2}}\right\}
\end{aligned}
$$

which depends only on the symmetric parts $\pi^{(i j)}$ and $e_{(i j)}$. Using the same procedure that we have used to obtain (9) from (8), we can write the reduced hamiltonian (31) in terms of spin-2 projection operators as

$$
H^{(r)}=\int \mathrm{d}^{(D-1)} x\left\{\pi^{(i j)}\left(P_{S S}^{(2)}\right)^{i j, k l} \pi^{(k l)}+e_{(i j)}\left(-\frac{\nabla^{2}}{4} P_{S S}^{(2)}\right)^{i j, k l} e_{(k l)}\right\},
$$

where we have used the constraints $\bar{\phi}=0$ and (29) to obtain the following relations

$$
\begin{aligned}
& \pi^{(i j)}\left(P_{S W}^{(0)}+P_{W S}^{(0)}\right)^{i j, k l} \pi^{(k l)}=2 \sqrt{D-2} \pi^{(i j)}\left(P_{W W}^{(0)}\right)^{i j, k l} \pi^{(k l)}, \\
& \pi^{(i j)}\left(P_{S S}^{(0)}\right)^{i j, k l} \pi^{(k l)}=(D-2) \pi^{(i j)}\left(P_{W W}^{(0)}\right)^{i j, k l} \pi^{(k l)} .
\end{aligned}
$$

Expression (32) is exactly the same one obtained in the massless FP theory (9).

\subsection{The $m \neq 0$ case}

The lagrangian density of the massive version of the $\mathcal{L}_{\mathrm{nFP}}$ in $D$ dimensions is given by [27]

$$
\mathcal{L}_{\mathrm{nFP}}^{m}=\mathcal{L}_{\mathrm{nFP}}-\frac{m^{2}}{4}\left(e_{\mu \nu} \mathrm{e}^{\nu \mu}+c e^{2}\right),
$$

\footnotetext{
${ }^{1}$ Note that the equation $(30)$ is a curl, so there are $(D-2)$ secondary constraints in $\chi^{[i j]}=0$.
} 
where $c$ is an arbitrary real constant. The massive case breaks the linearized reparametrization $\delta e_{\mu \nu}=\partial_{\nu} \xi_{\mu}$. The Weyl symmetry is also broken in general by the mass term except if we choose $c=-1 / D$.

The primary constraints are the same ones of the massless case. They are given by (23)-(25). The massive primary hamiltonian is

$$
H_{P}^{m}=\int \mathrm{d}^{D-1} x\left\{\mathcal{H}_{\mathrm{nFP}}^{m}+\lambda_{\mu 0} \phi^{\mu 0}+\lambda_{[i j]} \phi^{[i j]}+\bar{\lambda} \bar{\phi}\right\},
$$

where

$\mathcal{H}_{\mathrm{nFP}}^{m}=\mathcal{H}_{\mathrm{nFP}}+\frac{m^{2}}{4}(c+1) e_{00} e_{00}-\frac{m^{2}}{2} e_{0 i} e_{i 0}+\frac{m^{2}}{4} e_{i j} e_{j i}+\frac{m^{2} c}{4} \bar{e}\left(\bar{e}-2 e_{00}\right)$,

with $\mathcal{H}_{\mathrm{nFP}}$ given by (26).

From the consistency condition of the primary constraints (23)-(25) we find respectively

$$
\begin{aligned}
& \chi^{0}=\partial_{i} \pi^{0 i}+\left[\frac{\nabla^{2}}{2(D-1)}+\frac{m^{2}}{2} c\right] \bar{e}-\frac{m^{2}}{2}(1+c) e_{00} \approx 0, \\
& \chi^{i}=\partial_{j} \pi^{(i j)}+\frac{1}{4} \frac{(D-3)}{(D-1)} \partial_{i} \partial_{j} e_{0 j}-\frac{1}{4}\left(\nabla^{2}-2 m^{2}\right) e_{0 i} \approx 0, \\
& \chi^{[i j]}=\partial_{i}\left[\pi^{0 j}+\frac{1}{2} \partial_{k} e_{(k j)}\right]-\partial_{j}\left[\pi^{0 i}+\frac{1}{2} \partial_{k} e_{(k i)}\right]+m^{2} e_{[i j]} \approx 0, \\
& \psi^{0}=\frac{m^{2}}{2}(c D+1)\left(e_{00}-\bar{e}\right) \approx 0 .
\end{aligned}
$$

The equation $\dot{\chi}^{[i j]} \approx 0$ allows us to find $\lambda_{[i j]}$ while $\dot{\chi}^{0} \approx 0$ and $\dot{\psi}^{0} \approx 0$ furnish the multipliers $\lambda_{00}$ and $\bar{\lambda}$. On the other hand, $\dot{\chi}^{i} \approx 0$ leads to the tertiary constraints

$$
\zeta^{i}=-\frac{(D-1)}{(D-2)} m^{2} \pi^{0 i}-\frac{1}{2} \frac{(D-1)}{(D-2)} m^{2} \partial_{j} e_{(i j)}+\frac{m^{2}}{2} \partial_{i} \bar{e} \approx 0
$$

From $\dot{\zeta}^{i} \approx 0$ we have quaternary constraints

$$
\Omega^{i}=\frac{1}{2} \frac{(D-1)}{(D-2)} m^{4}\left(e_{0 i}-e_{i 0}\right) \approx 0 .
$$

The condition $\dot{\Omega}^{i} \approx 0$ determines the multipliers $\lambda_{i 0}$ and the procedure finally stops. All the Lagrange multipliers are determined if $c \neq-1 / D$, because in this case the model does not have any local symmetry. Therefore, the massive $\mathcal{L}_{\mathrm{nFP}}$ model has $D^{2}+D+2$ second class constraints and the total number degrees of freedom is $(D+1)(D-2) / 2$.

If we choose $c=-1 / D$, the $\mathcal{L}_{\mathrm{nFP}}(c=-1 / D)$ theory acquires Weyl symmetry. In this case the constraint $\psi^{0} \approx 0$, see (41), does not exist anymore. From $\dot{\chi}^{0} \approx 0$ we can determine, for instance, $\lambda_{00}$ in terms of $\bar{\lambda}$ while $\bar{\lambda}$ remains undetermined and appears in the Hamiltonian multiplying the combination $\phi^{0}-\bar{\phi}$. Thus, instead of the four second class constraints $\left(\phi^{00}, \bar{\phi}, \chi^{0}, \psi^{0}\right) \approx(0,0,0,0)$ we have one first class constraint $\phi^{0}-\bar{\phi} \approx 0$ and two second class ones that we can choose as $\chi^{0} \approx 0$ and $\phi^{00} \approx 0$. The total number of physical degrees of freedom remains the same in the Weyl invariant massive case $c=-1 / D$. The partially reduced hamiltonian is given by 


$$
\begin{aligned}
H^{(p r)}= & \int \mathrm{d}^{D-1} x\left\{\pi^{(i j)} \pi^{(i j)}+\frac{1}{3} \frac{(D-2)}{(D-1)} \bar{\pi} \bar{\pi}+\frac{1}{2} \frac{(D-3)^{2}}{(D-1)^{2}} \frac{\partial_{i} \bar{\pi} \nabla^{2} \partial_{i} \bar{\pi}}{\left(\nabla^{2}-2 m^{2}\right)^{2}}\right. \\
& +\frac{2(D-3)}{(D-1)} \frac{\partial_{i} \bar{\pi} \nabla^{2} \partial_{k} \pi^{i k}}{\left(\nabla^{2}-2 m^{2}\right)^{2}}+\frac{2 \partial_{i} \pi^{(i j)} \nabla^{2} \partial_{k} \pi^{(k j)}}{\left(\nabla^{2}-2 m^{2}\right)^{2}}-\frac{1}{4} \partial_{i} e_{(j k)} \partial_{i} e_{(j k)} \\
& \left.-\frac{1}{4} \partial_{i} \bar{e}\left[\partial_{i} \bar{e}-2 \partial_{k} e_{i k}\right]-\frac{1}{2} \partial_{i} e_{(i j)} \partial_{k} e_{(k j)}+\frac{m^{2}}{4} e_{(i j)} e_{(i j)}-\frac{m^{2}}{4} e_{i i} e_{j j}\right\} .
\end{aligned}
$$

In terms of the spin-2 projection operators we obtain the reduced Hamiltonian below given in terms of decoupled spin helicities

$$
\begin{gathered}
H^{(r)}=\int \mathrm{d}^{D-1} x\left\{\pi^{(i j)}\left[P_{S S}^{(2)}+\frac{4 m^{2}\left(m^{2}-\nabla^{2}\right)}{\left(\nabla^{2}-2 m^{2}\right)^{2}} P_{S S}^{(1)}+\frac{(D-1)^{3} m^{4} P_{W W}^{(0)}}{(D-2)\left[\nabla^{2}-(D-1) m^{2}\right]^{2}}\right]^{i j, k l} \pi^{(k l)}\right. \\
\left.+e_{(i j)}\left[\frac{\left(m^{2}-\nabla^{2}\right)}{4} P_{S S}^{(2)}+\frac{m^{2}}{4} P_{S S}^{(1)}+\frac{(D-1)}{4}\left(m^{2}-\nabla^{2}\right) P_{S S}^{(0)}\right]^{i j, k l} e_{(k l)}\right\}
\end{gathered}
$$

where we have used the following relations derived from the constraints

$$
\begin{aligned}
& \pi^{(i j)}\left(P_{S W}^{(0)}+P_{W S}^{(0)}\right)^{i j, k l} \pi^{(k l)}=\frac{2\left[(D-2) \nabla^{2}+(D-1) m^{2}\right]}{\sqrt{D-2}\left[\nabla^{2}-(D-1) m^{2}\right]} \pi^{(i j)}\left(P_{W W}^{(0)}\right)^{i j, k l} \pi^{(k l)}, \\
& \pi^{(i j)}\left(P_{S S}^{(0)}\right)^{i j, k l} \pi^{(k l)}=\frac{\left[(D-2) \nabla^{2}+(D-1) m^{2}\right]^{2}}{(D-2)\left[\nabla^{2}-(D-1) m^{2}\right]^{2}} \pi^{(i j)}\left(P_{W W}^{(0)}\right)^{i j, k l} \pi^{(k l)}, \\
& e_{(i j)}\left(P_{S W}^{(0)}+P_{W S}^{(0)}\right)^{i j, k l} e_{(k l)}=2 \sqrt{D-2} \frac{\left(\nabla^{2}-m^{2}\right)}{m^{2}} e_{(i j)}\left(P_{S S}^{(0)}\right)^{i j, k l} e_{(k l)} .
\end{aligned}
$$

Once again, based on (10) and the fact that the eigenvalues of $-\nabla^{2}$ are positive, the contribution to the Hamiltonian of each spin mode is positive definite. Up to overall factors, which can be canceled by field redefinitions, the Hamiltonians (17) and (45) are equivalent.

\section{The $\mathcal{L}_{a_{1}}$ model}

The next family of massive spin- 2 models we call $\mathcal{L}_{a_{1}}$, it depends on a real parameter $a_{1}$ present in the kinetic term, the mass term is of the usual FP form. At the special value $a_{1}=-1 / 4$ it has first appeared in [18] while for arbitrary values of $a_{1}$ it has appeared in [17], both in $D=4$. For the $D$-dimensional generalization see [27]. The model reduces to the usual FP theory at $a_{1}=1 / 4$. At $a_{1}=(3-D) /[4(D-1)]$ it coincides with the $\mathcal{L}_{\mathrm{nFP}}$ model of previous section at $c=-1$.

\subsection{The $m=0$ case}

As in the previous sections we first consider the massless theory in $D$-dimensions [27]

$$
\begin{aligned}
\mathcal{L}_{a_{1}}= & -\frac{1}{4} \partial_{\mu} e_{(\alpha \beta)} \partial^{\mu} \mathrm{e}^{(\alpha \beta)}+\frac{1}{2}\left(a_{1}+\frac{1}{4}\right) \partial_{\mu} e\left[\partial^{\mu} e-2 \partial_{\nu} \mathrm{e}^{\nu \mu}\right]+\frac{1}{2}\left[\partial_{\mu} \mathrm{e}^{(\mu \nu)}\right]^{2} \\
& +\frac{1}{2}\left(a_{1}-\frac{1}{4}\right)\left[\partial_{\mu} \mathrm{e}^{\mu \nu}\right]^{2} .
\end{aligned}
$$


It is invariant under the vector transformations $\delta e_{\mu \nu}=\partial_{\nu} \xi_{\mu}$ for arbitrary values of $a_{1}$. At $a_{1}=1 / 4$ it becomes also invariant under arbitrary antisymmetric shifts $\delta e_{\mu \nu}=\Lambda_{\mu \nu}=-\Lambda_{\nu \mu}$. If $a_{1}=-(D-3) / 4(D-1)$, see previous section, we have a larger set of symmetries: $\delta e_{\mu \nu}=\partial_{\nu} \xi_{\mu}+\eta_{\mu \nu} \Phi+\partial^{\alpha} \Lambda_{\alpha \mu \nu}$ where $\Lambda_{\alpha \mu \nu}$ is a totally antisymmetric tensor.

The massless version of $\mathcal{L}_{a_{1}}$ describes, if $a_{1} \neq 1 / 4$ and $a_{1} \neq(3-D) /[4(D-1)]$, massless spin-2 particles plus massless scalars (spin-0). The scalar theory is unitary if

$$
a_{1} \leqslant-\frac{D-3}{4(D-1)} \quad \text { or } \quad a_{1} \geqslant \frac{1}{4}
$$

Separating time and space components in the lagrangian density (47), we write the result as (up to total derivatives)

$$
\begin{aligned}
\mathcal{L}_{a_{1}}= & \frac{1}{2}\left(a_{1}-\frac{1}{4}\right) \dot{e}_{0 i} \dot{e}_{0 i}+\frac{1}{4} \dot{e}_{(i j)} \dot{e}_{(i j)}-\frac{1}{2}\left(a_{1}+\frac{1}{4}\right) \dot{\bar{e}} \dot{\bar{e}}+2\left(a_{1}+\frac{1}{4}\right) \dot{\bar{e}} \partial_{j} e_{(0 j)} \\
& -\dot{e}_{0 i}\left[\left(a_{1}-\frac{1}{4}\right) \partial_{i} e_{00}+a_{1} \partial_{j} e_{j i}+\frac{1}{4} \partial_{j} e_{i j}\right]-\frac{1}{2} \dot{e}_{(i j)} \partial_{i} e_{j 0}-\mathcal{V}
\end{aligned}
$$

where

$$
\begin{aligned}
\mathcal{V}= & -\frac{1}{2}\left(a_{1}-\frac{1}{4}\right) \partial_{i} e_{00} \partial_{i} e_{00}-\left(a_{1}+\frac{1}{4}\right) \partial_{i} e_{00}\left[\partial_{i} \bar{e}-\partial_{j} e_{i j}\right]+\frac{1}{2}\left(a_{1}+\frac{1}{4}\right) \partial_{i} \bar{e}\left[\partial_{i} \bar{e}-2 \partial_{j} e_{i j}\right] \\
& +\frac{1}{2} \partial_{i} e_{(0 j)} \partial_{i} e_{(0 j)}-\frac{1}{4} \partial_{i} e_{(j k)} \partial_{i} e_{(j k)}-\frac{a_{1}}{2} \partial_{i} e_{i 0} \partial_{j} e_{j 0}-\frac{1}{4} \partial_{i} e_{i 0} \partial_{j} e_{0 j}-\frac{1}{8} \partial_{i} e_{0 i} \partial_{j} e_{0 j} \\
& +\frac{a_{1}}{2} \partial_{i} e_{i j} \partial_{k} e_{k j}+\frac{1}{4} \partial_{i} e_{i j} \partial_{k} e_{j k}+\frac{1}{8} \partial_{i} e_{j i} \partial_{k} e_{j k} .
\end{aligned}
$$

The canonical momenta are given by

$$
\begin{aligned}
& \pi^{\mu 0}=0, \quad \pi^{[i j]}=0 \\
& \pi^{0 i}=\left(a_{1}-\frac{1}{4}\right)\left[\dot{e}_{0 i}-\partial_{i} e_{00}\right]-a_{1} \partial_{j} e_{j i}-\frac{1}{4} \partial_{j} e_{i j}, \\
& \pi^{(i j)}=\frac{1}{2} \dot{e}_{(i j)}-\left(a_{1}+\frac{1}{4}\right) \delta_{i j}\left[\dot{\bar{e}}-2 \partial_{k} e_{(0 k)}\right]-\frac{1}{2} \partial_{i} e_{j 0}-\frac{1}{2} \partial_{j} e_{i 0} .
\end{aligned}
$$

Thus, we have the following primary constraints in the massless version

$$
\begin{aligned}
& \varphi^{\mu 0}=\pi^{\mu 0} \approx 0, \\
& \varphi^{[i j]}=\pi^{[i j]} \approx 0 .
\end{aligned}
$$

The canonical Hamiltonian density can be written as 


$$
\begin{aligned}
\mathcal{H}_{a_{1}}= & \pi^{\mu} \dot{e}_{\mu \nu}-\mathcal{L}_{a_{1}} \\
= & \pi^{(i j)} \pi^{(i j)}+\frac{\pi^{0 i} \pi^{0 i}}{2\left(a_{1}-\frac{1}{4}\right)}-\left(a_{1}+\frac{1}{4}\right) \frac{\bar{\pi} \bar{\pi}}{C}+\left(a_{1}+\frac{1}{4}\right) \frac{\bar{\pi} \partial_{j} e_{0 j}}{C}+\pi^{(i j)} \partial_{i} e_{j 0} \\
& +\pi^{0 i}\left[\partial_{i} e_{00}+\frac{a_{1} \partial_{j} e_{j i}+\frac{1}{4} \partial_{j} e_{i j}}{\left(a_{1}-\frac{1}{4}\right)}\right]-\left[\frac{(D-1)}{2 C}\left(a_{1}+\frac{1}{4}\right)^{2}-\frac{1}{8}\right] \partial_{i} e_{0 i} \partial_{j} e_{0 j} \\
& -a_{1} \partial_{i} e_{0 i} \partial_{j} e_{j 0}-\frac{1}{8} \partial_{i} e_{0 j} \partial_{i} e_{0 j}-\frac{1}{4} \partial_{i} e_{0 j} \partial_{i} e_{j 0}+\frac{1}{4} \partial_{i} e_{(j k)} \partial_{i} e_{(j k)}+\frac{a_{1} \partial_{i} e_{i j} \partial_{k} e_{k j}}{8\left(a_{1}-\frac{1}{4}\right)} \\
& \left.+\frac{\partial_{i} e_{i j} \partial_{k} e_{j k}}{16\left(a_{1}-\frac{1}{4}\right)}-\frac{1}{8\left(a_{1}-\frac{1}{2}\right)} \partial_{1}-\frac{1}{4}\right)
\end{aligned}
$$

where $C$ is defined by

$$
C=\left[a_{1}(D-1)+\frac{D-3}{4}\right] .
$$

We are assuming that $a_{1} \neq 1 / 4$ and $C \neq 0$, since they correspond to models already treated in previous sections. The primary Hamiltonian is given by

$$
H_{P}=\int \mathrm{d}^{D-1} x\left[\mathcal{H}_{a_{1}}(x)+\lambda_{\mu 0}(x) \varphi^{\mu 0}(x)+\lambda_{[i j]}(x) \varphi^{[i j]}(x)\right],
$$

where $\lambda_{\mu 0}$ and $\lambda_{[i j]}$ are Lagrange multipliers.

Using (57) we obtain from the consistency condition (conservation in time) of the primary constraints the following secondary constraints

$$
\begin{aligned}
& \chi^{0}=\partial_{i} \pi^{0 i}+\left(a_{1}+\frac{1}{4}\right) \nabla^{2} \bar{e} \approx 0, \\
& \chi^{i}=\partial_{j} \pi^{(i j)}-a_{1} \partial_{i} \partial_{j} e_{0 j}-\frac{1}{4} \nabla^{2} e_{0 i} \approx 0, \\
& \chi^{[i j]}=\partial_{i}\left[\pi^{0 j}+\frac{1}{2} \partial_{k} e_{(k j)}\right]-\partial_{j}\left[\pi^{0 i}+\frac{1}{2} \partial_{k} e_{(k i)}\right] \approx 0 .
\end{aligned}
$$

Applying the consistency condition to the secondary constraint again we verify that are no tertiary constraints, and the Dirac-Bergmann algorithm finishes. Therefore, the massless $\mathcal{L}_{a_{1}}$ model has altogether $\left(D^{2}+3 D-2\right) / 2$ constraints, all first class. The Lagrange multipliers $\lambda_{\mu 0}$ and $\lambda_{[i j]}$ remain arbitrary. Thus, the number of the degrees of freedom is $(D-1)$ $(D-2) / 2$. In $D=4$ dimensions we have three degrees of freedom. It is in agreement with the observation in [27] that the massless version of $\mathcal{L}_{a_{1}}$ describes massless spin-2 particles plus a massless scalar field which disappears at $a_{1}=1 / 4$ and $a_{1}=-(D-3) / 4(D-1)$.

Using the constraints we can write down the Hamiltonian in a partially reduced form 


$$
\begin{aligned}
H^{(p r)}= & \int \mathrm{d}^{D-1} x\left\{\pi^{(i j)} \pi^{(i j)}-\left(a_{1}+\frac{1}{4}\right) \frac{\bar{\pi} \bar{\pi}}{C}-\frac{\partial_{i} \pi^{j j} \partial_{k} \pi^{i k}}{C \nabla^{2}}+\frac{2 \partial_{i} \pi^{(i j)} \partial_{k} \pi^{(j k)}}{\nabla^{2}}\right. \\
& +2\left[(D-1) a_{1}-\frac{1}{2}\right] \frac{\left[\partial_{i} \partial_{j} \pi^{i j}\right]^{2}}{C \nabla^{4}}+\frac{1}{4} \partial_{i} e_{(j k)} \partial_{i} e_{(j k)}+\frac{1}{4} \frac{\left(a_{1}+\frac{1}{4}\right)}{\left(a_{1}-\frac{1}{4}\right)} \partial_{i} e_{j j}\left[\partial_{i} e_{k k}-2 \partial_{k} e_{i k}\right] \\
& \left.-\frac{1}{2} \partial_{i} e_{(i j)} \partial_{k} e_{(k j)}-\frac{a_{1}}{2\left(a_{1}-\frac{1}{4}\right)} \frac{\left[\partial_{i} \partial_{j} e_{i j}\right]^{2}}{\nabla^{2}}\right\}
\end{aligned}
$$

The positivity of the Hamiltonian can be easily verified, since

$$
H^{(r)}=\int \mathrm{d}^{D-1} x\left\{\mathcal{H}_{s=2}+\mathcal{H}_{s=0}\right\},
$$

where

$$
\begin{gathered}
\mathcal{H}_{s=2}=\pi^{(i j)}\left(P_{S S}^{(2)}\right)^{i j, k l} \pi^{(k l)}-\frac{1}{4} e_{(i j)}\left(\nabla^{2} P_{S S}^{(2)}\right)^{i j, k l} e_{(k l)}, \\
\mathcal{H}_{s=0}=\frac{\left(a_{1}-1 / 4\right)}{C(D-2)}\left\{\left[\theta_{i j}-(D-2) \omega_{i j}\right] \pi^{(i j)}\right\}^{2}-\frac{C}{4\left(a_{1}-1 / 4\right)} e_{(i j)}\left(\nabla^{2} P_{S S}^{(0)}\right)^{i j, k l} e_{(k l)} .
\end{gathered}
$$

We have a spin- 2 and a spin- 0 massless particle. The spin- 0 Hamiltonian can be brought to a canonical form $\mathcal{H}_{s=0}=\left(\tilde{\pi}^{2}-\tilde{e} \nabla^{2} \tilde{e}\right) / 2$ after the change of variables

$$
\tilde{e}=\sqrt{\frac{2}{C(D-2)}} \theta^{i j} e_{i j} \quad ; \quad \tilde{\pi}=\sqrt{\frac{C}{2(D-2)}}\left[\theta^{k l}-(D-2) \omega^{k l}\right] \pi_{k l} .
$$

where $\{\tilde{e}, \tilde{\pi}\}=1$ follows from the canonical brackets between $e_{i j}$ and $\pi_{k l}$. Remember that in the massless case we have no second class constraints before gauge fixing. The spin- 2 contribution (63) is always positive definite while the spin-0 one (64) is positive whenever the unitarity conditions (48) are satisfied. This is in full agreement with the Lagrangian analysis of [17].

\subsection{The $m \neq 0$ case}

The massive $\mathcal{L}_{a_{1}}$ lagrangian density in $D$ dimensions is given by [27]

$$
\mathcal{L}_{a_{1}}^{m}\left(e_{\alpha \beta}\right)=\mathcal{L}_{a_{1}}\left(e_{\alpha \beta}\right)-\frac{m^{2}}{4}\left(e_{\mu \nu} \mathrm{e}^{\nu \mu}-e^{2}\right) .
$$

The mass term breaks the symmetry under linearized reparametrization $\delta e_{\mu \nu}=\partial_{\nu} \xi_{\mu}$ but does not affect the primary constraints (53) and (54). The primary Hamiltonian becomes

$$
H_{P}^{m}=\int \mathrm{d}^{D-1} x\left[\mathcal{H}_{a_{1}}^{m}(x)+\lambda_{\mu 0}(x) \varphi^{\mu 0}(x)+\lambda_{[j]}(x) \varphi^{[i j]}(x)\right],
$$

where the canonical Hamiltonian density $\mathcal{H}_{a_{1}}^{m}$ is

$$
\mathcal{H}_{a_{1}}^{m}=\mathcal{H}_{a_{1}}-\frac{m^{2}}{2} e_{0 i} e_{i 0}+\frac{m^{2}}{4} e_{i j} e_{j i}-\frac{m^{2}}{4} \bar{e}\left(\bar{e}-2 e_{00}\right),
$$

and $\mathcal{H}_{a_{1}}$ is given by (55). 
Applying the Dirac-Bergmann algorithm, from $\dot{\Pi}^{\mu 0} \approx 0$ and $\dot{\Pi}^{[i j]} \approx 0$ we find the secondary constraints

$$
\begin{aligned}
& \chi^{0}=\partial_{i} \pi^{0 i}+\left[\left(a_{1}+\frac{1}{4}\right) \nabla^{2}-\frac{m^{2}}{2}\right] \bar{e} \approx 0, \\
& \chi^{i}=\partial_{j} \pi^{(i j)}-a_{1} \partial_{i} \partial_{j} e_{0 j}-\frac{1}{4}\left(\nabla^{2}-2 m^{2}\right) e_{0 i} \approx 0, \\
& \chi^{[i j]}=\partial_{i}\left[\pi^{0 j}+\frac{1}{2} \partial_{k} e_{(k j)}\right]-\partial_{j}\left[\pi^{0 i}+\frac{1}{2} \partial_{k} e_{(k i)}\right]+m^{2} e_{[i j]} \approx 0 .
\end{aligned}
$$

The consistency condition $\dot{\chi}^{[i j]} \approx 0$ gives the multiplier $\lambda_{[i j]}$ while $\dot{\chi}^{0} \approx 0$ and $\dot{\chi}^{i} \approx 0$ lead to the following tertiary constraints respectively

$$
\begin{aligned}
& \zeta^{0}=\frac{m^{2} \bar{\pi}}{2 C}-\frac{m^{2}(D-1)}{2 C}\left(a_{1}+\frac{1}{4}\right) \partial_{i} e_{0 i} \approx 0, \\
& \zeta^{i}=\frac{m^{2}}{\left(a_{1}-\frac{1}{4}\right)} \pi^{0 i}+\frac{m^{2}}{2\left(a_{1}-\frac{1}{4}\right)} \partial_{j} e_{(i j)}+m^{2} \partial_{i} \bar{e} \approx 0 .
\end{aligned}
$$

Going on, from $\dot{\zeta}^{0} \approx 0$ and $\dot{\zeta}^{i} \approx 0$ we find the respective quaternary constraints

$$
\begin{aligned}
& \Omega^{0}=\frac{m^{4}(D-1)}{4 C}\left(-e_{00}+\bar{e}\right) \approx 0, \\
& \Omega^{i}=\frac{m^{4}}{2\left(a_{1}-\frac{1}{4}\right)}\left(e_{i 0}-e_{0 i}\right) \approx 0 .
\end{aligned}
$$

Last, $\dot{\Omega}^{0} \approx 0$ and $\dot{\Omega}^{i} \approx 0$ furnish $\lambda_{00}$ and $\lambda_{i 0}$, and the procedure finishes up. Therefore, there are $D^{2}+D+2$ constraints in the massive $\mathcal{L}_{a 1}$ model. They are all of second class and it is possible to determine all the multipliers $\lambda_{00}, \lambda_{i 0}$ and $\lambda_{[i j]}$. The number of degrees of freedom is $(D+1)(D-2) / 2$ in agreement with the massive FP and massive nFP models.

Using the constraints we can write the partially reduced hamiltonian for the massive $\mathcal{L}_{a 1}$ as

$$
\begin{aligned}
H^{(p r)}= & \int \mathrm{d}^{D-1} x\left\{\pi^{(i j)} \pi^{(i j)}-\frac{\left[8(D-1) a_{1}\left(a_{1}+\frac{1}{2}\right)+\frac{(D-3)}{2}\right]}{8(D-1)^{2}\left(a_{1}+\frac{1}{4}\right)^{2}} \bar{\pi} \bar{\pi}+\frac{2 \partial_{i} \pi^{(i j)} \nabla^{2} \partial_{k} \pi^{(k j)}}{\left(\nabla^{2}-2 m^{2}\right)^{2}}\right. \\
& +\frac{2 a_{1} \partial_{i} \bar{\pi} \nabla^{2}}{(D-1)^{2}\left(a_{2}+\frac{1}{4}\right)^{2}} \frac{\left[a_{1} \partial_{i} \bar{\pi}-2(D-1)\left(a_{1}+\frac{1}{4}\right) \partial_{k} \pi^{i k}\right]}{\left(\nabla^{2}-2 m^{2}\right)^{2}}+\frac{1}{4} \partial_{i} e_{(j k)} \partial_{i} e_{(j k)} \\
& \left.-\frac{1}{4} \partial_{i} \bar{e}\left[\partial_{i} \bar{e}-2 \partial_{k} e_{k i}\right]+\frac{1}{2} \partial_{i} e_{(i j)} \partial_{k} e_{(k j)}+\frac{m^{2}}{4} e_{(i j)} e_{(i j)}-\frac{m^{2}}{4} \bar{e}^{2}\right\} .
\end{aligned}
$$

The reduced hamiltonian is given by

$$
H^{(r)}=\int \mathrm{d}^{D-1} x\left\{\mathcal{H}_{s=2}+\mathcal{H}_{s=1}+\mathcal{H}_{s=0}\right\}
$$


where

$$
\begin{aligned}
& \mathcal{H}_{s=2}=\pi^{(i j)}\left(P_{S S}^{(2)}\right)^{i j, k l} \pi^{(k l)}+\frac{1}{4} e_{(i j)}\left[\left(m^{2}-\nabla^{2}\right) P_{S S}^{(2)}\right]^{i j, k l} e_{(k l)}, \\
& \mathcal{H}_{s=1}=\frac{4 m^{2}}{\left(\nabla^{2}-2 m^{2}\right)^{2}} \pi^{(i j)}\left[\left(m^{2}-\nabla^{2}\right) P_{S S}^{(1)}\right]^{i j, k l} \pi^{(k l)}+\frac{m^{2}}{4} e_{(i j)}\left(P_{S S}^{(1)}\right)^{i j, k l} e_{(k l)}, \\
& \mathcal{H}_{s=0}=\frac{m^{4} \bar{\pi}\left(1 / \nabla^{4}\right) \bar{\pi}}{(D-1)(D-2)\left(a_{1}+\frac{1}{4}\right)^{2}}+\frac{(D-1)}{4} e_{(i j)}\left[\left(m^{2}-\nabla^{2}\right) P_{S S}^{(0)}\right]^{i j, k l} e_{(k l)} .
\end{aligned}
$$

The constraints have been used to eliminate redundant degrees of freedom and contractions with the operators $P_{W W}^{(0)}$ and $P_{S W}^{(0)}+P_{W S}^{(0)}$. As in the previous massive models, the spin-2, spin-1 and spin- 0 contributions are separately positive. For sake of comparison with (17) and (45) we are tempted to rewrite the first term of (80) in terms of contractions with $P_{W W}^{(0)}$. By using the constraints $\zeta^{0} \approx 0$ and $\partial_{i} \chi^{i} \approx 0$ we can deduce

$$
\partial_{i} \partial_{j} \pi^{i j}=\frac{\left[\left(a_{1}+1 / 4\right) \nabla^{2}-m^{2} / 2\right]}{(D-1)\left(a_{1}+1 / 4\right)} \bar{\pi}
$$

We can eliminate $\bar{\pi}$ in terms of $\partial_{i} \partial_{j} \pi^{i j}$ and get

$$
\frac{m^{4} \bar{\pi}\left(1 / \nabla^{4}\right) \bar{\pi}}{(D-1)(D-2)\left(a_{1}+\frac{1}{4}\right)^{2}}=\pi^{(i j)}\left\{\frac{m^{4}(D-1)\left(P_{W W}^{(0)}\right)_{i j k l}}{(D-2)\left[\left(a_{1}+1 / 4\right) \nabla^{2}-m^{2}\right]^{2}}\right\} \pi^{(k l)} .
$$

The apparently singular point $\left(a_{1}+1 / 4\right) \nabla^{2}-m^{2}=0$, is not a true singularity as we see from the left hand side of (82). From (81) it is clear that $\pi^{(i j)}\left(P_{W W}^{(0)}\right)_{i j k} \pi^{(k l)}$ vanishes at such point which by the way only appears if $a_{1}<-1 / 4$ (outside the unitarity domain of the corresponding massless theory).

\section{Conclusion}

Here we have applied the plain Dirac-Bergmann algorithm in three known families of second order (in derivatives) theories describing massive spin-2 particles via a rank-2 tensor. In section 2 we have reviewed the well known case of the massless and massive FP theory while in sections 3 and 4 we have investigated the more recent nFP and $\mathcal{L}\left(a_{1}\right)$ families respectively. Although there is no local field redefinition relating those models, they all describe, if $m \neq 0$, massive spin-2 particles in a unitary way. We have proved explicitly that their reduced Hamiltonian $H^{(r)}$ depends only on the symmetric components of a rank-2 tensor $e_{(\mu \nu)}$ and their momenta $\pi_{(\mu \nu)}$. The number of constraints leads to the correct counting of degrees of freedom which fits massive spin-2 particles in $D=4$. In the $\mathrm{nFP}$ case, see (35), there is a special point ( $c=-1 / 4)$ where the whole massive theory acquires Weyl symmetry, but we still have the correct number of degrees of freedom thanks to the fact that one second class constraint disappears while another one becomes first class. Our main result is that $H^{(r)}$ is positive definite in both $\mathrm{nFP}$ and $\mathcal{L}\left(a_{1}\right)$ cases for arbitrary values of their free parameters $c$ and $a_{1}$ respectively. In particular, each spin-2, spin- 1 and spin- 0 mode contributes with a positive factor separately. This is in agreement with the analytic properties of the propagator in both theories as examined in [17]. 
Regarding the massless theories, the reduced Hamiltonian $H^{(r)}$ of the massless nFP model coincides with the Hamiltonian of the massless FP theory (linearized Einstein-Hilbert), they carry only spin- 2 modes ( +2 and -2 helicities). The reduced Hamiltonian $H^{(r)}$ of the massless version of $\mathcal{L}\left(a_{1}\right)$ reveals that we have spin- 2 altogether with spin-0 propagating modes in the theory. Such massless scalar-tensor theory is unitary whenever the conditions (48) are satisfied in agreement with the Lagrangian analysis of [17].

We conclude that the free spin-2 theories $\mathcal{L}_{\mathrm{nFP}}(c)$ and $\mathcal{L}\left(a_{1}\right)$ are on the same footing of the paradigmatic FP theory also from the Hamiltonian point of view which indicates that such theories might deserve some attention regarding the development of self-interacting spin-2 theories (massive and massless). Since the Hamiltonian approach has proved to be instrumental in developing new massive gravity theories, in particular, in proving absence of ghosts, see e.g. chapter 7 of [9], the analysis made here is a first step in future developments.

\section{Acknowledgments}

DD thanks Prof Odylio Aguiar for bringing [2] to his attention, he also thanks CNPq (307278/2013-1) for financial support. AR dos S thanks UNESP for the financial support. DB thanks CAPES for the financial support.

\section{Appendix}

The spin- 0 and spin-1 projection operators acting on vector fields in $D$ dimensions are defined in the spacial sector by

$$
\omega_{i j}=\frac{\partial_{i} \partial_{j}}{\nabla^{2}} \quad, \quad \theta_{i j}=\delta_{i j}-\omega_{i j}
$$

respectively. Using those operators one can define the projection and transition symmetric operators

$$
\begin{aligned}
& \left(P_{S S}^{(2)}\right)^{i j, k l}=\frac{1}{2}\left(\theta^{i k} \theta^{j l}+\theta^{i l} \theta^{j k}\right)-\frac{\theta^{i j} \theta^{k l}}{D-2}, \\
& \left(P_{S S}^{(1)}\right)^{i j, k l}=\frac{1}{2}\left(\theta^{i k} \omega^{j l}+\theta^{i l} \omega^{j k}+\theta^{j k} \omega^{i l}+\theta^{j l} \omega^{i k}\right), \\
& \left(P_{S S}^{(0)}\right)^{i j, k l}=\frac{\theta^{i j} \theta^{k l}}{D-2}, \quad\left(P_{W W}^{(0)}\right)^{i j, k l}=\omega^{i j} \omega^{k l}, \\
& \left(P_{S W}^{(0)}\right)^{i j, k l}=\frac{\theta^{i j} \omega^{k l}}{\sqrt{D-2}}, \quad\left(P_{W S}^{(0)}\right)^{i j, k l}=\frac{\omega^{i j} \theta^{k l}}{\sqrt{D-2}},
\end{aligned}
$$

and they satisfy the symmetric closure relation

$$
\left[P_{S S}^{(2)}+P_{S S}^{(1)}+P_{S S}^{(0)}+P_{W W}^{(0)}\right]^{i j, k l}=\frac{1}{2}\left(\delta^{i k} \delta^{j l}+\delta^{i l} \delta^{j k}\right) .
$$

The previous operators satisfy the algebra ${ }^{2}$

\footnotetext{
${ }^{2}$ In $D=3+1$ the projection operators $P_{J K}^{(s)}$ represent spin-s operators, since the trace $11_{\mu \nu, \alpha \beta}\left(P_{J K}^{(s)}\right)^{\mu \nu, \alpha \beta}$ is $2 s+1$, where $11_{\mu \nu, \alpha \beta}=\left(\eta_{\mu \alpha} \eta_{\nu \beta}+\eta_{\mu \beta} \eta_{\nu \alpha}\right) / 2$. In the present work we deal with $D-1$ spatial indices, we replace $\eta_{\mu \nu}$ by $\delta_{i j}$, however we keep calling $P_{J K}^{(s)}$, abusively, spin-s operators.
} 


$$
P_{I J}^{(r)} P_{K L}^{(s)}=\delta^{r s} \delta_{J K} P_{I L}^{(r)},
$$

where $I, J, K, L=S, W$ and $r, s=0,1,2$.

Consider a second order Hamiltonian density in $D$ dimensions, whose spacial sector is given by

$\mathcal{H}=-\frac{s_{1}}{2} \partial_{i} f_{j k} \partial_{i} f_{j k}-\frac{s_{2}}{2} \partial_{i} \bar{f} \partial_{i} \bar{f}-s_{3} \partial_{i} \bar{f} \partial_{j} f_{i j}-s_{4} \partial_{i} f_{i j} \partial_{k} f_{k j}+\frac{s_{5}}{2} f_{i j} f_{i j}+\frac{s_{6}}{2} \bar{f} \bar{f}$,

where $s_{i}$ are real free parameters and $f_{i j}$ a symmetric tensor field with $\bar{f}=\delta_{i j} f_{i j}$. This hamiltonian density can be written as

$$
\mathcal{H}=\frac{1}{2} f_{i j} \mathcal{O}^{i j, k l} f_{k l},
$$

where, suppressing the indices, the differential operator is given by

$$
\begin{aligned}
\mathcal{O}= & {\left[s_{1} \nabla^{2}+s_{5}\right] P_{S S}^{(2)}+\left[\left(s_{1}+s_{4}\right) \nabla^{2}+s_{5}\right] P_{S S}^{(1)}+\left[\left(s_{1}+(D-2) s_{2}\right) \nabla^{2}+s_{5}+(D-2) s_{6}\right] P_{S S}^{(0)} } \\
& +\left[\left(s_{1}+s_{2}+2 s_{3}+s_{4}\right) \nabla^{2}+s_{5}+s_{6}\right] P_{W W}^{(0)}+\sqrt{D-2}\left[\left(s_{2}+s_{3}\right) \nabla^{2}+s_{6}\right]\left(P_{S W}^{(0)}+P_{W S}^{(0)}\right) .
\end{aligned}
$$

\section{References}

[1] The LIGO Scientific Collaboration and The Virgo Collaboration 2016 Phys. Rev. Lett. 116061102

[2] The LIGO Scientific Collaboration and The Virgo Collaboration 2016 Phys. Rev. Lett. 116221101

[3] van Dam H and Veltman M J G 1970 Nucl.Phys. B 22 397-411

[4] Zakharov V I 1970 JETP Lett. (Sov. Phys.) 12312

[5] Boulware D G and Deser S 1972 Phys. Lett. B 40 227-9

[6] Vainshtein A I 1972 Phys. Lett. B 39393

[7] Babichev E and Deffayet C 2013 Class. Quantum Grav. 30184001

[8] Hinterbichler K 2012 Rev. Mod. Phys. 84 671-710

[9] de Rham C 2014 Massive gravity Living Rev. Relativ. 177

[10] Riess A G et al 1998 Astron. J. 1161009

[11] Perlmutter S et al 1999 Astrophys. J. 517565

[12] de Rham C, Gabadadze G and Tolley A J 2011 Phys. Rev. Lett. 106231101

[13] Hassan S and Rosen R A 2012 J. High Energy Phys. JHEP02(2012)126

[14] Fierz M and Pauli W 1939 Proc. R. Soc. A 173 211-32

[15] Koenigstein A, Giacosa F and Rischke D H 2016 Ann. Phys. 368 16-55

[16] Bonifacio J, Ferreira P G and Hinterbichler K 2015 Phys. Rev. D 91125008

[17] Dalmazi D 2013 Phys. Rev. D 87125027

[18] Morand K and Solodukhin S N 2012 Phys. Lett. B 715 260-6

[19] Hassan S and Rosen R A 2012 Phys. Rev. Lett. 108041101

[20] Hassan S F and Rosen R A 2012 J. High Energy Phys. JHEP04(2012)123

[21] Dirac P A M 1964 Lectures on Quantum Mechanics (New York: Yeshiva University)

[22] Sundermeyer K 1982 Constrained Dynamics (Berlin: Springer)

[23] Hanson A, Regge T and Teitelboim C 1976 Constrained Hamiltonian Systems (Roma: Accademia Nazionale dei Lincei)

[24] Marzban C, Whiting B F and van Dam H 1989 J. Math. Phys. 301877

[25] Deser S 2015 Can. J. Phys. 93 395-7

[26] Dalmazi D 2012 Phys. Rev. D 86125036

[27] Dalmazi D, dos Santos A L R and Mendona E L 2015 Ann. Phys., NY 354 385-93

[28] Casini H, Montemayor R and Urrutia L F 2002 Phys.Rev. D 66085018 